\title{
Commentaires sportifs en direct et présent psychologique
}

\author{
Mustapha Krazem ${ }^{1}$ \\ Université de Lorraine, Metz, CREM, France
}

\begin{abstract}
Résumé. mon but est de réfléchir à la façon dont les commentaires sportifs en direct construisent leur système temporel : présent de l'indicatif, interjections, phrases averbale, futur etc. J'insisterai sur les différences que fait Wilmet sur trois présents : présent physique, présent psychologique et présent linguistique. Le présent psychologique est l'élément important pour appréhender la temporalité des commentaires sportifs. Les exemples proviennent d'enregistrements en direct télé ou radio, effectués à l'occasion de championnats de football et des jeux olympiques de Pékin, Londres et Rio.
\end{abstract}

\begin{abstract}
My goal is to think about how in french sports live commentary constructs his time system : «présent de l'indicatif », interjection, « futur de l'indicatif », sentence without conjugated verb. I will renforce the importance of Marc Wilmet for my study because this linguist differentiates three presents » : physical present, psychological present and linguistic present. Sports commentary needs psychological présent to be studied. The examples come from several sports recorded live on television and radio during football championships and during the Beijing, London and Rio Olympics
\end{abstract}

\section{Introduction}

Depuis quelques années de nombreuses études de grammaire de la phrase intègrent plus ou moins fortement l'objet "genre de discours » tel qu'il a été introduit par Bakhtine (1984). Cette intégration est souvent minimale, pour diversifier l'origine des données. Elle peut cependant être plus poussée avec pour but de décrire plus précisément le fonctionnement du système ${ }^{1}$. Le recours méthodologique aux genres de discours est plus ancien en sémantique verbale. En attestent des travaux comme ceux de Bres (2005) ou Wilmet (1980, 1997). Le confirmeront si besoin les nombreux tiroirs du présent de l'indicatif étiquetés du genre de discours (ou situation discursive) où on les repère : présent scénique, présent de narration, présent des titres, présent pictural, présent des histoires drôles...De plus, on sait depuis longtemps, que les types de texte, ont des dispositifs temporels contraints. Les narrations utilisent bien volontiers des passés simples tout en ignorant l'impératif. Aussi, ce qui a été bien étudié pour les genres littéraires peut être étendu à n'importe quel genre de discours, comme l'a fait Adam (2001) avec les genres procéduraux. C'est dans cette optique que je me propose de décrire la temporalité dans les commentaires sportifs en direct. Plus exactement, seront décrits tous les éléments linguistiques, dont le système verbal, permettant au commentateur de situer la description de l'événement par rapport au présent physique. Le genre « commentaire sportif », on s'en doute, n'est pas pris au hasard. C'est un champ approprié pour participer à la description théorique de l'objet « genre de discours ». Il utilise des supports variés : radio, télé, presse ; il est réactif aux nouvelles technologies ; il a connu des évolutions majeures depuis plus d'un siècle d'existence ; mais surtout c'est un des rares genres de discours qui s'élabore en temps réel, « en direct».

Nul doute que c'est cette caractéristique qui a contribué à populariser les retransmissions sportives. L'attrait du public pour des sports à grande intensité a même eu des conséquences sur les règles. Plusieurs sports ont adapté leurs règles de fonctionnement pour permettre des phases d'intensité, donc de commentaires en direct et ainsi conduire à un renforcement de l'intérêt médiatique. C'est le cas du ski de fond et du biathlon. D'autres, pour lesquels les phases d'intensité étaient impossibles à multiplier ont imaginé des moyens originaux pour retenir l'attention du public : c'est le cas de la formule 1 avec des caméras embarquées ou encore du cyclisme, dans les retransmissions duquel une part importante est accordée à l'antenne à la description des monuments rencontrés sur les parcours. Incontestablement, le commentaire en direct a quelque chose à voir avec le succès médiatique des spectacles sportifs.

C'est la dimension linguistique dont il sera question ici. Le commentaire sportif " en direct » a pour caractéristique majeure de témoigner de la langue permise lorsque la parole doit être rapidement réalisée. On peut aller jusqu'à inférer que ce commentaire est un observatoire possible du rapport entre la langue et la perception du monde. N'étant pas spécialiste de linguistique cognitive, je ne m'aventurerai pas sur ce terrain-là. Je me contenterai de décrire le système en m'appuyant sur des exemples variés puisés dans les

\footnotetext{
${ }^{1}$ mustapha.krazem@univ-lorraine.fr
} 
commentaires issus de plusieurs sports, football principalement, enregistrés à la télé et à la radio, parfois simultanément ${ }^{2}$. J'insisterai, dans la deuxième partie de la contribution, sur ce qui structure crucialement la temporalité de ces commentaires en direct : le rapport au présent psychologique tel que Wilmet (1980) l'a intégré à la réflexion linguistique. Il en découlera les parties suivantes où seront traités successivement les marqueurs de procès dans la zone du présent psychologique et les expressions temporelles des procès situés antérieurement et postérieurement à l'action présente. Mais avant, je commencerai par préciser ce qu'il faut entendre par commentaires « en direct».

\section{Que faut-il entendre par commentaires sportifs en direct?}

Mais que faut-il entendre par commentaires sportifs en direct ? Comment ce sous-genre des commentaires sportifs doit- il être délimité ? On se doit de répondre à une double nécessité :

a-Celle de mettre en lien l'étiquetage ${ }^{3}$ du genre avec la réalité des productions prises en compte. Un locuteur lambda doit être en mesure de distinguer ce qui est un match commenté en direct, même s'il n'a aucun goût pour les spectacles sportifs.

b- C'elle de circonscrire cette réalité avec les objectifs linguistiques que le linguiste se donne. Le positionnement adopté ici est celui de Krazem (2011a). Le niveau d'analyse le plus utile à la description grammaticale est celui des sous- genres, voire des sous-sous genres. Cette seconde nécessité explique le filtre qu'il faut appliquer à la perception des matchs commentés en direct. Ne sont pris en compte que les moments de réels commentaires des actions sportives. Les discussions entre commentateurs, les miniinterviews ou autres paroles hors actions sportives n'ont aucune raison d'être intégrées à une étude s'intéressant au rapport entre la production de langage et un événement concomitant.

Cependant, même une fois délimités ainsi les CS en direct restent partiellement hétérogènes. Il existe des phases dynamiques, intenses et par contraste des phases plus calmes. Le choix de certains sports pour illustrer mon propos est parfois lié à cette variation dans le rythme du commentaire. Celui d'une prestation de gymnastique est dynamique dans son intégralité. C'est bien moins fréquent dans le commentaire d'une course cycliste sur route. D'autres sports, comme le football et le rugby, alternent de façon égale les phases dynamiques et statiques.

Les commentaires radio et télé ne seront pas toujours distingués sauf dans les exemples. Il existe certes des différences linguistiques du fait de la présence de l'image. Mais il m'a semblé intéressant de traiter unitairement les deux moyens de diffusion parce qu'ils participent au même objectif de quête du présent physique. Il sera cependant mis en valeur les variations pertinentes. C'est ainsi que ce que j'appellerai plus loin « le principe de continuité du commentaire radio » permet d'étendre la zone du présent par l'emploi de temps du passé, passant dans la même séquence du commentaire en direct au commentaire immédiatement différé.

Quel que soit le sport, le commentateur a toujours en tête de rester en lien avec le présent physique, le présent de l'action sportive. Pour cela, à la radio, et dans une moindre mesure à la télévision, le journaliste sportif use d'éléments linguistiques dont la fonction est de réajuster le commentaire sur le présent physique comme c'est observé dans Grinshpun/Krazem (2005). Cette opération de réajustement peut être pleinement formulée par une phrase du type « on revient sur le jeu ». Le plus souvent, on a recours à d'autres procédés, comme les adverbes « là » ou « maintenant» ou encore la conjonction « alors que » et la préposition « avec $»$.

(1) Barthez qui avait anticipé peut-être un peu la frappe de Tomer était pas super bien placé sur la frappe de Morera mais il ne pouvait pas grand-chose Les Lensois égalisent et les Marseillais sont retombés dans leurs travers/ Et là Varouzec sur le coté/ Avec un ballon bien dégagé en touche par Fanny/ (foot multiplexe radio)

(2) On est maintenant à une quarantaine de mètres (foot multiplexe radio)

(3) Avec une longue touche là effectué par les Lensois (foot multiplexe radio)

Le commentaire sportif en direct dont le système temporel sera décrit est donc cette partie du commentaire dont la motivation est la description immédiate des actions perçues par le commentateur. Intuitivement, on pense d'emblée au présent de l'indicatif. Néanmoins la suite de cette contribution montrera d'une part, que linguistiquement, la captation d'un procès au présent peut s'opérer de plusieurs façons ; d'autre part, qu'il existe aussi des contraintes temporelles dans l'expression de l'ultérieur et de l'antérieur de ce moment présent, contraintes qui sont calculées par rapport au présent des actions de jeu. Il est donc nécessaire, particulièrement pour notre genre de discours, de réfléchir à qui renvoie le terme " présent ». C'est ce que je ferai en suivant Marc Wilmet. 


\section{Les trois présents de Wilmet}

Je ne m'arrêterai pas ici sur les théories du présent de l'indicatif ${ }^{4}$ mais sur les distinctions opérées par Marc Wilmet (1980) entre plusieurs « présents ». Il me semble qu'indépendemment des choix théoriques que l'on peut faire dans la description de ce temps, il est difficile de faire l'économie des décalages temporels qui s'ensuivent des trois présents, comme vont le montrer plus loin les commentaires en direct.

\subsection{La mise en évidence du présent psychologique}

Parmi toutes les avancées théoriques que le linguiste belge a apportées figure indéniablement sa distinction entre trois présents là où l'intuition des non-spécialistes n'en voit qu'un : les présents physique, psychologique et linguistique. Comme cela apparaîtra dans la citation ci-dessous, Wilmet propose de ne pas traiter le « présent » de façon globale car cette globalité masque des réalités distinctes, trois visions qui gagnent à être isolées. Le présent physique est le présent réel, valable pour les être humains comme pour l'univers. Le temps linguistique appelé " présent » est intuitivement (et abusivement comme on le sait) amalgamé à ce présent physique. A ces deux présents, Wilmet montre la pertinence d'un présent psychologique, qui renvoie à la perception par le locuteur des événements du présent physique.

«-Le présent des physiciens : en déplacement perpétuel, insaisissable »

-Le présent des psychologues. Etat « de conscience » (Bergson) ou temps « privé » (Russel) : soit l'intervalle objectif - variant de zéro à plusieurs secondes au gré des circonstances extérieures et de l'attention des sujets- qui sépare deux stimuli perçus comme simultanés.

- Le présent des linguistes. Rapport de concomitance institué entre acte de langage (renonciation) et un événement (l'énoncé) grâce à divers « mots temporels » : maintenant, actuel, (Pierre) marche »

Cette distinction s'avère particulièrement éclairante lorsqu'il s'agit des commentaires sportifs. Le présent physique (le

« vrai » présent) n'a pas d'épaisseur. C'est un curseur qui avance inéluctablement, transformant en passé le futur qu'il rencontre. Le passé et le futur, eux, ont une épaisseur. Il est possible d'y concevoir des successions : deux procès au passé ou au futur qui se suivent restent dans le passé ou le futur tandis que le premier de deux procès qui se suivent au présent devient passé au moment de la réalisation du second. Du moins au présent physique. Ce qui est ne sera plus et laisse la place à ce qui est et qui ne sera plus et ainsi de suite.

Le présent linguistique est la forme verbale à laquelle on apparie généralement l'expression du présent physique. On restera ici au seul présent de l'indicatif ${ }^{5}$. Nous verrons très vite (\$3.1) que lorsque le présent sportif est invoqué par les chercheurs pour appuyer une des trois thèses mentionnées à la note $\mathrm{n}^{\circ} 4$, la pertinence du présent psychologique est occultée. Ce dernier est la nouveauté introduite par Wilmet. Le présent psychologique introduit de plein pied le locuteur dans la description temporelle : par sa perception de l'événement, et, j'ajouterai, par tout ce qu'un locuteur est susceptible d'introduire ou de ressentir dans un énoncé : sentiment, recul, ironie... J'ajouterai encore, par la mise en résonnance de l'action à l'instant présent avec la durée de l'énonciation de la langue qui formule cette action. La notion de présent psychologique peut ainsi déborder largement du présent de l'indicatif et éclairer la réalisation par un locuteur de n'importe quel procès oral ou écrit, quel qu'en soit le tiroir verbal.

Contrairement au présent physique, le présent psychologique a une durée, durée qui peut varier. Or, cette variabilité, et les commentaires sportifs le montrent, n'est pas une constante unique, mais est plutôt une zone de réaction. Il en découle une conséquence majeure : le présent psychologique, comme le passé et le futur, a uneépaisseur.

\subsection{Epaisseur et point de captation}

C'est dans cette épaisseur du présent psychologique que vont fleurir différents procédés linguistiques, procédés qui s'étalent par captations ${ }^{6}$ successives. La suite de l'article décrira, exemplifiera et justifiera l'ordre de ces captations en fonction du repérage du présent physique mais cet ordre peut déjà être annoncé. Le procédé le plus proche du présent physique sans jamais l'atteindre est l'interjection «oh », puis arrivent dans l'ordre «ah », l'allongement vocalique, les trois types d'énoncés averbaux. Cette zone se referme sur le présent de l'indicatif, ce qui explique très vraisemblablement une partie des discussions sur son caractère accompli ou déictique. Directement en lien avec le présent psychologique, deux temps permettent au commentateur d'exprimer l'antérieur : le passé composé et, curieusement, lorsqu'il s'agit de clore une séquence, le futur simple. Enfin, l'expression de l'ultérieur ne prend qu'une seule forme : le présent prospectif (ou futur périphrastique), et depuis quelque temps, l'infinitif lorsqu'il s'agit de procès virtuels. Un point de méthode doit être exposé maintenant. Pour déterminer l'ordre des captations, outre des arguments linguistiques qui seront présentés à leur place, j'insiste sur l'importance du recul permis par l'examen a posteriori des occurrences avec le support. Particulièrement à la télévision. C'est ce recul qui rend visible cet ordre. J'admets cependant qu'une quantification plus précise affinerait ma proposition de 
classement.

A côté de cette zone du commentaire en direct, il existe une autre zone certes proche mais qui ne doit pas être confondue : celle du « présent psychologique étendu » (Krazem 2011a) : ralenti, replay, multiplexe radio, voire le direct écrit par internet. Cette zone ne sera pas étudiée spécifiquement dans cette contribution. On en trouvera des éclairages dans Krazem (2005 et 2011a). Elle fournira cependant ici des points de contraste avec les formes en vrai direct.

\section{Les marqueurs de procès dans la zone du présent psychologique}

Les procès de la zone du présent psychologique sont marqués par plusieurs procédés, verbaux ou non, procédés plus ou moins proches du présent physique. L'expression des actions sportives au « présent » ne se contente pas du " présent de l'indicatif ». Ce n'est même pas le procédé majoritaire. Avant de présenter ces procédés du plus proche du présent physique au plus lointain, il est utile d'effectuer un détour par les exemples utilisés qui ont été choisis antérieurement par les chercheurs lorsqu'ils sollicitent le présent sportif pour décrire le présent en général. La non-prise en compte des réelles conditions discursives et l'omission du paramètre du présent psychologique expliqueront l'irréalisme plus ou moins marqué des exemples présentés. Cependant, qu'on n'y voit aucun mépris de ma part car cet irréalisme a permis aussi de construire des hypothèses pertinentes.

\subsection{L'inadéquation des exemples des études antérieures}

Les travaux traitant du présent de l'indicatif, immanquablement, accordent un passage aux commentaires sportifs en direct sans, toutefois, que ces commentaires soient décrits pour eux-mêmes. C'est toujours le rapport au présent physique qui motive les exemples sportifs. Or, le présent psychologique n'est pas sollicité dans les descriptions. De ce fait, il est facile de constater a posteriori un manque de réalisme avec les exemples construits proposés et la réalité du corpus. On s'en convaincra par un simple coup d'œil comparatif entre la série (5), provenant de travaux bien connus, avec la retranscription attestée (6) :

(5a) Et c'est Lola Montes, une jument de trois ans, qui passe la ligne d'arrivée la première ! (Nef 1986)

(5b) Laffite franchit la ligne. (Bonnard 1982)

(5c) Dubois intercepte la balle, feinte le troisième ligne gallois Smith, mais l'arrière Baker surgit et

l'accroche...Dubois passe à Dupont qui marque entre les poteaux. (Serbat 1980)

(5d) X passe la Balle à Y. Y passe la balle à Z, qui tire et qui marque. (Gosselin 2000) (5e) Platini tire au but...hé non! il perd sa chaussure (Martin 1987)

(5f) la porte s'ouvre. Le ministre sort de la maison. (il porte un costume rouge). Il traverse le jardin et monte dans sa voiture. (Co Vet 1980)

(6) Et là Back qui tourne sur le côté/ avec Lachor / Le pressing de Johansen / Lachor/ qui a joué le long de la ligne pour Bakari / En retrait pour Coridon / Bon petit contrôle / La faute peut-être de Meité / Voilà c'est sifflé / C'est un coup franc côté gauche et Lachor qui va le tirer / Lachor qui joue en profondeur... (direct radio)

La comparaison entre (5) et (6) appelle plusieurs remarques.

(i) Plusieurs de ces exemples (5a et b) ont pour but de montrer que le présent de l'indicatif capte des faits déjà accomplis. J'y reviendrai plus loin.

(ii) Les exemples (5) sont des phrases canoniquement bien formées. Or, une caractéristique du corpus est la quantité très importante d'énoncés averbaux, lesquels captent plus précocement le moment de l'action.

(iii) De toutes façons, le présent sportif a du mal à être formaté par une phrase complète, ne serait-ce que parce que la mobilité inéluctable interfère avec la durée de l'énonciation. On s'en apercevra plus loin avec les structures de la forme

« Nom du joueur + QUI relative »

(iv) La présence de tous les actants, au point que Gosselin (5d) les remplace par des variables, dépend du média. C'est vrai à la radio. La télé se contente le plus souvent du nom du joueur seul.

(v) Des marques linguistiques temporelles non verbales contribuent à repérer les procès par rapport au moment actuel. Ce fait est annoncé dans Wilmet (1980). C'est le cas des énoncés averbaux ou des interjections « oh » et « ah ». Quant à l'interjection « hé » de l'exemple de (5e), non seulement elle ne s'observe pas en direct mais on peut affirmer que (5e) ne peut être que la formulation d'une plaisanterie du genre « vidéo gag ». Pour énoncer (5e), il faut être au courant de la perte de chaussure de Platini ! Et avoir le projet d'en plaisanter!

(vi) L'intonation et le rythme ne sont pas pris en compte. Pourtant, plusieurs emplois temporels trouvent 
une explication meilleure lorsqu'on accorde une place aux faits prosodiques, notamment les faits d'allongement vocalique. On doit aussi tenir compte de la délimitation des séquences énoncés, lesquelles montrent que la langue est produite par noyaux prédicatifs successifs. Mes transcriptions, à cet égard, apparaîtront minimales (pose d'un slash démarquant les noyaux) mais on se référera avantageusement aux travaux de Mathon/Boulakia (2011), mieux documentés sur ce point.

(vii) L'exemple (5c) de Serbat n'est pas attestable en direct, ne serait-ce que quand on imagine le temps nécessaire aux procès décrits et la bien longue phrase qui les commente ! Cette phrase ne serait envisageable que de façon nettement différée. Il est, entre autres, impossible en direct de réaliser une ellipse du pronom sujet dans ces conditions-là. Il en va de même pour la conjonction « et ». Pour être réalisée, il lui faut un recul, une vision à plus long terme des procès, ce qui est incompatible avec un commentaire en direct en phase dynamique. Enfin, ce qui est possible dans le contexte de l'exemple (5f) ne l'est pas avec les commentaires sportifs. Cet exemple est inadapté à être assimilé au présent sportif puisqu'il en perd une part importante de la substance.

Il ressort de cet examen rapide de (5) que ces exemples ne correspondent pas à ce qui est observé en réalité. Les commentaires en direct proposent peu de phrases complètes, ce qui réduit de facto l'emploi du présent de l'indicatif. Et, nous le verrons, quand celui-ci est employé, c'est souvent dans une relative continuative ou à la voix passive. L'organisation des commentaires s'apparente bien davantage à une suite de groupes prédicatifs juxtaposés avec une intonation marquée. Enfin, la transcription linguistique des procès perçus par le commentateur ne recourt pas uniquement à des verbes à temps fini (présent ou passé composé). D’autres procédés linguistiques permettent des captations plus proches du présent physique, captations successives dont il est question à partir d'ici.

\subsection{Première captation oh/ah}

La première captation linguistique du procès dans le présent psychologique est celle opérée par l'interjection «oh ». Grinshpun/Krazem (2005) en décrivent le comportement dans les commentaires sportifs où elle est fréquente. On ne la trouve qu'en direct, toujours dans des situations extrêmement saillantes (7). Il arrive parfois que le procès justifiant l'interjection ne soit perçu que lors du « ralenti » (8).

(7a) Et corner ici pour Châteauroux, oh il est bien tiré oh il est superbe le troisième but de Châteauroux. (multiplexe radio)

(7b) Oh l'intervention de Fabien Barthez (télé, coupe du monde 98)

(7c) Batistuta ! en position...oh ! Et premier but (télé, coupe du monde 98)

(8) (sur un coup franc) Oh que c'est bien fait! il y est! C'est toute la Corée du sud qui se lève ! (en commentant le ralenti qui suit) Oh détourné le ballon ! détourné par un défenseur ! (télé, coupe du monde 98)

L'antériorité de la captation de « oh » par rapport à l'ensemble des autres marqueurs est sensible dans la mesure où cette interjection est toujours placée devant l'énoncé auquel elle se rapporte. Dans la même perspective, il est inconcevable que « oh » soit énoncée longtemps (i.e quelques secondes) après un procès, contrairement aux autres procédés.

(7a)* Il est très bien tiré oh, il est superbe le troisième but de Châteauroux oh ! (7b)*L'intervention de Fabien Barthez oh !

$(7 \mathrm{c})^{*}$ Batistuta ! en position... Et premier but ! oh !

L'interjection « Oh » ne marque pas le début de l'action qu'il précède. Elle est quasi simultanée au procès exprimé par l'énoncé qui suit et qui explicite son emploi. Un petit décalage apparaît à l'observation attentive du corpus télévisé, décalage qui confirme le temps psychologique de Wilmet. Ainsi, dans l'exemple (7a), lorsque l'énonciateur commence son énoncé, il ne sait pas encore que le but va avoir lieu. $\mathrm{Au}$ fur et à mesure de la verbalisation de son énonciation, la balle s'approche du but. La fin de l'énoncé coïncide avec le but marqué par le joueur. Et c'est là où surgit le second oh. L'interjection « ah » relève également de cette première captation bien qu'elle soit très légèrement postérieure.

Grinshpun/Krazem (2005) montrent que «ah » intègre un point de vue préalable, absent de « oh ». Pour « oh », il n'y a pas expression d'un point de vue au sens de Rabatel (1998). Il s'agit pour l'énonciateur de valider l'état de choses par lequel il est «dominé » : aucun autre point de vue n'est envisagé. En revanche, lorsque c'est « ah » qui est utilisé, la construction de l'énoncé implique d'une part que l'énonciateur valide un état de choses existant, mais aussi qu'il envisage un autre point de vue. L'opération cognitive engageant l'énonciation de «ah » est de facto un peu plus longue.

(9) Attention il a Rotten dans son dos ! ah il choisit la solution axiale et malheureusement il reperd le ballon. (direct radio) 
(10) Attention à cette attaque des Bastiais/ avec une balle dans la surface de réparation pour Tony Verrelle/ ah il a du mal à tenir debout tellement ça glisse sur ce terrain. (direct radio)

\subsection{Deuxième captation les allongements vocaliques}

Dans les commentaires en direct, plus que dans n'importe quel autre genre de discours, la durée de l'énonciation peut interférer sur la syntaxe et sur la temporalité. La trace la plus originale en est l'allongement vocalique, qui mime la durée d'une action. Avec ce procédé, bien connu des auditeurs, le commentateur peut « choisir » (mais choisit-il vraiment ?) de faire correspondre la durée du procès (ou du moins la perception qu'il a du procès) avec l'énonciation. Il est fréquent d'entendre un allongement vocalique se maintenant le temps de la durée du procès, comme une sorte d'iconicité auditive. Dans l'exemple (11) reconstruit ci-dessous, l'allongement sur le « en » correspond à la course du ballon ${ }^{8}$.

(11) Et Zidane qui cennnnntre !

Les allongements s'opèrent généralement sur les termes constituant les procès. Mais pas nécessairement. Ainsi, dans l'exemple (12), la voyelle nasale (en majuscules) est allongée jusqu'à son interruption par le procès représenté par le groupe qui suit. L'allongement est indexé au curseur continu du présent physique tel que le perçoit le commentateur. L'exemple (12) ne donne pas une description dans la durée du «ballon » (voir aussi note $\mathrm{n}^{\circ} 10$ ) mais signifie qu'il se passe quelque chose de très important dans le présent perçu.

(12) Zidane qui donne un bon ballOON Henri maintenant sur le côté gauche (coupe du monde 1998)

\subsection{Troisième captation : les énoncés averbaux}

Les énoncés averbaux relèvent de la troisième captation. Ils sont massivement employés autant à la radio qu'à la télé. Ils restent importants dans "le présent psychologique étendu » (voir plus haut) mais commencent alors à davantage alterner avec le présent de l'indicatif.

On doit voir dans cette utilisation une conséquence des propriétés formelles des énoncés averbaux en général. D’une part, ils n’ont pas de « sujet » obligatoire. D’autre part, lorsque celui-ci apparaît, il suit le terme prédicatif (nom, adjectif ou participe passé). Le commentaire en direct favorisant la description de l'action, le procès est mis en avant au détriment de l'agent. On notera toutefois que le commentaire radio demandant davantage de précisions, la mention de l'agent y est généralement réalisée.

Les énoncés averbaux justifient d'être sous-catégorisés en trois ensembles aisément identifiables. Les termes techniques, qui représentent un procès sous forme nominale, ainsi que les passifs sans sujet. Le troisième ensemble est une originalité du commentaire sportif : l'emploi de noms propres prédicatifs ${ }^{9}$. Les deux premiers ensembles sont captés plus précocement que le troisième. Ils apparaissent dans des phases précises de jeu. Les noms propres ont une zone de captation moins contrainte car ils sont employés aussi largement dans les phases non dynamiques.

\subsubsection{Les termes techniques}

Ces termes constituent le gros bataillon des supports prédicatifs des phrases averbales. Même si cela n'a pas d'incidence sur la place dans la zone de captation du présent psychologique, on peut distinguer dans cet ensemble :

-les termes techniques purs : but, corner, uppercut, penalty, salto...Dans certains sports, certaines figures ont le nom des sportifs qui les ont inventées (gymnastique).

-les termes devenus techniques : sprint, centre, tir, mur, drop, ballon ${ }^{10}$. C'est parmi ces termes qu'on trouve le plus de nominalisations déverbales.

Comme la catégorie précédente, les conditions d'apparition dépendent des facteurs externes qui structurent le genre de l'extérieur. Le commentaire télévisé direct se contente souvent du terme seul puisque le visuel suffit souvent. Pour certains sports (gym, ski), il est parfaitement inutile de dire qui exécute le mouvement puisque le sportif a été identifié antérieurement. Le mouvement est vu, il suffit de le nommer (13):

(13) allez c'est parti / prise d'élan demi-tour à l'équilibre tout de suite et là ça accélère immédiatement pour un lâcher de barre / Kovacs plus vrille et un salto par-dessus la barre avec une vrille / on sent qu'il est parti avec une grosse envie/ beaucoup de rythme / dislocation / franchissement dorsale de barre tendu / figure du pigeon /tour volant plus demi-tour / Passage sur le dos /Et quatre tours/Il est sorti (gymnastique Jeux Olympiques Rio) 


\subsubsection{Les passifs}

Ce sous-ensemble des énoncés averbaux mérite d'être traité à part, d'une part par ce qu'il est assez fréquent et, d'autre part, parce qu'il est directement en lien avec une forme verbale. Comme le relève Brès (2007), à qui je renvoie pour une description plus précise, les passifs se limitent souvent au seul participe passé avec ou sans complément d'agent. Ce point me paraît être un argument pour considérer les passifs sans sujet comme relevant d'une analyse adjectivale plutôt que verbale. Cela étant, cela ne change rien à leur antériorité dans la perception par rapport au présent de l'indicatif puisque le procès (qu'il soit adjectival ou verbal) est directement énoncé.

(14a) Corner de Juninho au centre mais la balle est repoussée par les rennais/récupérée par Delfladre/qui s'infiltre sur le côté droit (multiplexe radio)

(14b) Oh détourné le ballon! détourné par un défenseur ! (télé, coupe du monde 98)

\subsubsection{Les noms propres.}

Une des caractéristiques fortes des commentaires sportifs en direct est l'usage prédicatif des noms propres. Pour Deulofeu (2000), l'énonciation du nom du joueur X signifie approximativement «X fait une action de jeu saillante ». Ces noms ne sont pas des étiquettes, et signifient sommairement « $\mathrm{X}$ joue » ou bien « $\mathrm{X}$ agit ». Je préciserai la description de Deulofeu, en ajoutant que l'action doit rester dans une dynamique qui n'est pas achevée. Un nom propre devient ainsi équivalent à la paraphrase (15).

(15) ZIDANE = «Zidane, en tant que joueur qui est en position, en lien avec le ballon, de poursuivre la dynamique du jeu ».

En effet, il est important que le jeu reste dans une dynamique continue, qui n'envisage pas de point d'achèvement, ce que reflète la fréquence importante des enchaînements avec " qui », sur lesquels je reviendrai. Toutefois, la phase de jeu n'est pas nécessairement dynamique. On différentie généralement les réalisations en phase dynamique par l'intonation plus marquée qui les accompagnent.

Quand l'action conduit à la fin d'une séquence de jeu, les commentateurs ne se servent pas du nom seul. Ils n'utilisent pas (ou très rarement) de nom de joueur lorsque celui-ci fait une faute qui arrête le jeu. Pas davantage lorsque le gardien de but fait un arrêt. Dans Krazem (2011b), j'explore linguistiquement ce fonctionnement et montre comment le nom propre cumule ainsi deux fonctions : prédicative ${ }^{11}$ et agentive. Cette double fonction épouse les objectifs d'un commentaire pour lequel agent et procès sont à mettre en avant.

(16) *Flamini / Avec Marley / Marley qui accélère /Qui accélère encore sur son côté

Steve Marley /qui est taclé par Carboni / Les marseillais vont récupérer ce ballon à la faveur de la touche / C'est joué vite Ferera avec Marley qui a du soutien / Flamini / Ferera / Marley/Flamini / Un ballon renvoyé par Marcena / Récupéré par N'Daye le bon retour la frappe de Ferera / Et la prise de balle de Canisares (télé direct)

Le paramètre visuel accentue la fréquence des noms propres seuls. A la radio, l'auditeur étant privé d'images, il est fréquent que la structure régisse une ou plusieurs relatives :

(17) Avec Barara / Robin Barara qui repique dans l'axe /qui va donner latéralement maintenant vers Vicente le buteur de Valence ce soir / qui rejoue là-bas de nouveau vers Couro Tores / Couro Tores qui monte / qui va pouvoir centrer peut-être / qui peut donner maintenant vers Rufete / (radio direct)

\subsection{Quatrième captation : Le présent de l'indicatif}

Le présent de l'indicatif s'avère être le procédé qui est capté le plus tardivement. On comprend de ce fait pourquoi de nombreux auteurs insistent sur l'aspect accompli de ces présents puisque le procès est déjà réalisé quand il est énoncé. C'est incontestablement vrai, du moins pour les procès qui nous intéressent ici. Cependant, c'est vrai aussi des interjections «oh/ah », des allongements vocaliques et des énoncés averbaux ! La question n'est donc pas de savoir si le présent de l'indicatif est accompli par rapport au présent physique mais s'il est accompli par rapport au présent psychologique. C'est très différent.

Or, indépendamment des théories descriptives du présent de l'indicatif, si les présents des phases dynamiques sont captés dans la zone du présent psychologique la plus lointaine du présent physique, ils restent encore perçus dans le présent psychologique. Dès que les procès cessent d'être perçus de la sorte alors arrivent des passés composés. A quoi serviraient alors les passés composés ici si le présent était accompli ?

La meilleure preuve, selon moi, de cette inscription dans la zone du présent psychologique est que si 
l'on peut concevoir des commentaires uniquement au présent de l'indicatif, on n'atteste aucun commentaire en direct qui ne serait qu'au passé composé. Soit ainsi la séquence (18), essentiellement au présent de l'indicatif ou au présent prospectif (ou futur périphrastique), futurs dont on notera qu'ils peuvent, pour la plupart, être remplacés par des présents. La transformation de l'intégralité de la séquence au passé composé est impossible. C'est possible partiellement, impossible totalement. Du moins dans les commentaires sportifs en direct. Seuls les présents de l'indicatif restent dans ce présent psychologique, y compris quand ils semblent être placés dans l'ultérieur (voir plus loin).

(18) Avec Barara/ Robin Barara qui repique dans l'axe/ qui va donner (donne) latéralement maintenant vers Vicente/ le buteur de Valence ce soir / qui rejoue là-bas de nouveau vers Cora Tores/ qui monte/ qui va pouvoir centrer peut-être/ qui peut donner maintenant vers Rufete

Rufete qui redonne à Couro Tores/ qui va déborder (déborde)/ qui va centrer (centre) en retrait et heureusement pour les marseillais aucun espagnol n'est resté aux abords de la surface de réparation

L'emploi du présent, avec son corollaire d'actants réalisés en position sujet à gauche, est confirmé par une forme particulièrement fréquente dans le commentaire radio : la forme " GN + qui » (Bres 2007, Krazem 2011b). Cette structure est assimilable à une relative continuative. C'est le cas dans l'exemple (18) ci-dessus. L'emploi de la relative introduite par « qui $»^{12}$ permet deux procès consécutifs : le premier porté par le nom propre, lequel devient sujet de la relative qui contient le second procès.

D'autres présents de l'indicatif se présentent sous une forme plus orthodoxe (Krazem 2005). L'exemple (19a) se situe à l'intérieur d'un procès perçu comme itératif. Quant à l'exemple (19b), c'est davantage une description d'un état (« il ne comprend pas ») qu'un réel procès. Il en est de même pour (19c).

(19a) Il enchaîne les tours de bras/travail proche de la barre/tour volant/à l'accélération/le fouetté/double vrille. (JO gymnastique télé)

(19b) Et l'ukrainien continue sur sa lancée/il ne comprend pas Ce qui lui arrive Kadafi/ il a été touché/il est sonné/il est compté/oh là là et c'est terminé ! (JO direct boxe)

(19c) Mitsa reste à terre. (foot direct radio)

Il ne faut pas déduite des exemples (18) et (19) que tous les présents de l'indicatif appartiennent à la zone du présent psychologique. Dès que l'on quitte le commentaire en direct, les présents de l'indicatif sont plus nombreux. Il est logique que le commentateur, connaissant l'événement et son aboutissement, ait le recul lui permettant de formuler une phrase complète. C'est confirmé par les commentaires en direct écrits sur internet, y compris dans des phases qui seraient dynamiques en direct (20) :

(20) BUT de Savidan pour Caen! Savigol libère le Stade Malherbe ! Quel geste de grande classe de l'international ! Après un long ballon de Sorbon, Savidan s'impose face à Faty et réalise un geste aérien du pied droit pour lober Richert impuissant! Quel sang froid! (match Caen-Sochaux internet orange)

\section{Antérieur et ultérieur}

Certes, le commentaire en direct, surtout dans ses phases rapides, tend à se rapprocher du présent physique. Cela n'exclut pas que ce commentaire puisse exprimer des procès antérieurement ou postérieurement à la zone du présent psychologique. On s'apercevra dans cette quatrième partie que les zones du passé et du futur sont très contraintes et montrent des procédés parfois intuitivement surprenants.

\subsection{La zone du passé}

Dans cette zone, les procès sont perçus et présentés en recul par rapport au présent physique. On sort de la zone du présent psychologique, en y restant dépendant. Le passé composé, véritable accompli du présent, sera le temps essentiel de cette zone, mais on repère parfois des imparfaits et des plus-que-parfaits. Ne seront abordés ici que les seuls passés composés en tentant de mieux saisir la différence d'emploi avec le présent de l'indicatif, dont les procès à l'aspect récent (venir de+infinitif). Sera abordé pour terminer l'emploi paradoxal du futur de l'indicatif comme marque d'antériorité.

\subsubsection{Les passés composés}

Nous venons de le voir, bien que le présent de l'indicatif soit apte à exprimer des procès antérieurs, il ne peut commuter totalement avec le passé composé dans les commentaires en direct. Partiellement oui, totalement non. On peut en déduire que les commentateurs ont ainsi recours au passé composé quand ils perçoivent l'action décrite comme étant achevée au commencement de l'énoncé.

Une explication supplémentaire concerne les directs à la radio. Dans Krazem (2005), par comparaison d'une même séquence en temps réel entre la télé et la radio, on constate ce que j'ai appelé «principe de 
continuité du commentaire radio ". Le commentaire radio, faute d'images, doit aider l'auditeur à se représenter mentalement la scène. Cela passe par une élaboration plus grande du discours. Le commentateur connaît assez souvent le dénouement de l'action qu'il retransmet parallèlement au moment où il est en train de produire son énoncé. Dans l'exemple (21) qui suit, comme l'atteste " avec là », l'action est repérée sur le moment actuel. L'action est déjà achevée et perçue comme telle par le commentateur au moment du tir («a tenté sa chance ») ${ }^{13}$ du joueur Moulida.

(21) Avec là une bonne balle à destination de Moulida / bien lancé par le jeune Renoir/ Moulida un peu excentré sur le côté droit de la surface de réparation// il a tenté sa chance / mais sans aucune difficulté pour le gardien.

\subsubsection{Les passés récents}

Lorsqu'on considère le sémantisme aspectuel de la forme périphrastique « venir de +inf » (Bonnard 1982), on s'attend logiquement à en trouver dans les commentaires en direct autant si ce n'est plus que des passés composés. Or, ces passés récents ne sont pas repérés dans le corpus en direct travaillé à l'occasion de cette contribution. Ils sont réalisés (22), mais toujours dans le présent psychologique étendu (ralenti, multiplexe, internet...).

(22a) Une frappe cadrée signée Essien à l'instant juste avant l'action que je viens de vous décrire (multiplexe radio) (22b) Florian Mayer, coupable de trois grosses fautes directes, vient de perdre huit points consécutivement (Internet tennis)

(22c)Oh le deuxième but ici à Montpellier/Stupeur au stade de la Mosson car c'est Daniel Cousin qui vient d'inscrire ce second but (multiplexe radio)

Cette absence de passé récent, qui mérite d'être davantage creusée, livre une piste intéressante. On sait que cette forme aspectuelle est étroitement dépendante d'un repérage : énonciatif en discours et relatif en récit, d'où le présent et l'imparfait comme seuls temps aptes à investir cette forme. De ce fait, son absence dans les commentaires en direct laisserait entendre que la position énonciative du commentateur, au moins dans les phases dynamiques, tend à disparaître, tout en réapparaissant dès qu'un recul, même minimal, est possible.

\subsubsection{Les futurs simples accomplis}

Une singularité du futur simple de l'indicatif dans les commentaires en direct est de n'être employé, à quelques exceptions près, que de façon accomplie, lorsqu'on connait l'aboutissement de l'action. Paradoxalement, les futurs ne sont possibles que lorsque l'action est passée (23). La commutation avec un passé composé est licite.

On se trouve alors devant une sorte de futur historique. D'une certaine façon, ce n'est pas surprenant puisque le commentaire bascule alors dans une sorte de séquence narrative.

(23) Voilà/ il joue vite Scacel/ qui essaie d'accélérer/ et aux prises là avec Jabi le défenseur Lensois/ et Jabi obtiendra un coup franc sifflé par Monsieur Guarigian. (foot direct radio).

Comme Bres (2007) le signale, certains futurs périphrastiques ou « présents prospectifs » peuvent avoir aussi une valeur accomplie. Toutefois, cela relève de la même explication que les présents intégrés dans la zone du présent psychologique dont il a été question plus haut. Nous allons le voir, ces présents prospectifs ont une fonction plus décisive dans l'expression de l'ultérieur.

\section{4 .2 La zone futur}

Le commentaire peut être amené à se projeter vers l'ultérieur. Lorsqu'on s'attarde sur ce point, on s'aperçoit que le champ du possible linguistique est très réduit. L'expression de l'ultérieur est pourtant quasiment une nécessité. Dans la mesure où un événement sportif contient une durée, des probabilités, des interruptions et des choix, le commentateur doit disposer de formes linguistiques appropriées. Or, le commentaire est aveugle à un avenir jamais scénarisé à l'avance. L'intérêt des matchs est qu'ils ont chacun un scénario improvisé et différent. Un commentaire doit donc anticiper des choix, des tactiques, des actions de jeu.

\subsubsection{Le nécessaire présent prospectif (futur périphrastique)}

Un fait remarquable apparaît immédiatement à l'observation : c'est le présent prospectif qui est seul 
nécessaire et suffisant pour exprimer l'ultérieur de la zone du présent psychologique. Il apparait que, ni le futur simple, ni le présent pro futuro, ne sont sollicités pour exprimer l'ultériorité dans les phases de commentaires dynamiques (24). Cela ne signifie pas que certains procès au présent de l'indicatif ne soient pas en réalité antérieurs à leur réalité physique, mais il ne s'agit pas pour autant de présent pro futuro. Ils ne font qu'anticiper leur matérialisation dans une perception dans le présent physique. Soit dit en passant, les exemples de Nef et Bonnard (rappelés ci-dessous), peuvent tout aussi bien être exprimés de façon anticipée et non une fois réalisée. Si un cheval a cinquante mètres d'avance à dix mètres du poteau d'arrivée, un présent est possible (mais pas encore un passé composé).

$(24 \mathrm{a}) *$ Il tirera bientôt $(24 \mathrm{~b}) *$ Il tire bientôt

(5a) Et c'est Lola Montes, une jument de trois ans, qui passe la ligne d'arrivée la première ! (Nef 1986)

(5b) Laffite franchit la ligne. (Bonnard 1982)

On vérifiera dans l'exemple radio direct (25) que l'emploi du futur simple est inadéquat. La substitution est possible au présent, mais avec des conséquences interprétatives, qui découlent d'analyses dont il a été fait état précédemment.

(25) Là c'est Auxerre qui a la balle sur le côté gauche avec Akalé qui va provoquer (*provoquera) son défenseur On essaie de trouver la tête de Akalé au deuxième poteau Mais attention le ballon est contré par Pesley qui a réussi à remettre ce ballon en retrait de la tête à Richer et là c'est un ballon qui est repris par Lachuer sur le côté droit qui va pouvoir donner (*donnera) dans l'axe à Akalé qui va revenir (*reviendra) sur son pied gauche il va rentrer (*rentrera) dans la surface de réparation il va tirer (*tirera) voilà un tir à ras de terre mais qui est stoppé par Richer/

L'absence du futur et du présent pro futuro s'explique parce que, indépendamment de leur valeur modale ${ }^{14}$, ils obligent une coupure temporelle, même minime. Or, une coupure suppose un repérage fixe à partir duquel il est possible de construire une distance et de concevoir des successions. Le commentaire sportif en direct ne permet pas la concrétisation de cette distance.

En dehors des futurs « historiques » dont j'ai parlé pour la zone du passé, les rares futurs de l'indicatif clairement repérables dans l'ultérieur physique sont associés à la reconstitution d'une coupure temporelle. On trouve ainsi des exemples du type (26), souvent interrogatifs.

(26a) Sortira-t-il le carton rouge ?

(26b) On espère que ce n'est pas trop grave et qu'il reviendra rapidement sur le terrain.

A contrario, le présent prospectif est sollicité pour sa propriété aspectuelle. Il maintient un lien direct avec le moment de l'énonciation, comme dans les exemples quelquefois cités pour l'opposer au futur simple

(27)Elle va avoir un enfant // Dépêche-toi, le train va partir !

\subsubsection{Le futur virtuel : les infinitifs dans les commentaires sportifs oraux}

En parallèle de l'expression de l'ultérieur, une forme verbale apparait, semble-t-il de plus en plus fréquemment : l'infinitif libre (27).

(27a) «Ne pas concéder de points avant de rentrer aux vestiaires !» (France-Angleterre, France 2,

01/2/14) (27b) «Allez ! Le libérer !» (Il s'agit du ballon en mêlée - Match France-Galles, TF1,

$16 / 10 / 12)$

(27c) Ne pas reculer, ne pas reculer! (match de boxe Khadafi conte un ukrainien, JO 2012)

Pourquoi en parallèle ? Parce qu'il ne s'agit pas véritablement de décrire l'événement à venir. C'est la manifestation d'une crainte ou de l'espoir d'un développement futur. De plus, cet événement virtuel est teinté d'une émotion qui est partagée par l'auditeur/téléspectateur. La description précise de ces infinitifs dépasse le cadre de cet article mais leur interprétation est du même ordre que celle repérée dans les titres de presse sportifs « du vendredi », c'est-à-dire du jour qui précède le week-end, moment où se déroulent la majorité des épreuves sportives. Les pages sportives utilisent volontiers l'infinitif avant l'événement (28). Le " sujet » de l'infinitif est toujours associé à l'équipe locale. Or, contrairement à l'intuition, ces infinitifs ne s'adressent pas aux joueurs. Avec l'infinitif, le journal transmet à ses lecteurs, dont le journal est complice, une sorte d'appropriation, d'incarnation, d'identification à l'équipe. Ces infinitifs ne sont donc pas injonctifs mais seraient plutôt sémantiquement, appellation provisoire, « exhortatif réflexif». 
(28b) Poursuivre sur le même chemin (Dauphiné du 24 aout 07) (28c) Eviter les calculs (La dépêche du midi 31 mars 07)

Dans les commentaires en direct, on ne les rencontre pas dans n'importe quel contexte : la vitesse du jeu, l'intensité de l'instant et le soutien à une des deux équipes, en l'occurrence celle des téléspectateurs. On est loin du « allez les petits » de Roger couderc ! Cependant, pareillement, ces infinitifs permettent de communier, de partager l'émotion avec les auditeurs/téléspectateurs ${ }^{15}$.

\section{Conclusion}

Cette contribution avait un double objectif. Décrire, dans sa globalité, le fonctionnement du système verbal (au sens large) des commentaires en direct. Ce genre de discours met en valeur un oral qui s'élabore sans recul par rapport au contenu exprimé, au point que souvent le terme d'une séquence n'est pas connu au début de son énonciation. J'ai insisté sur l'importance du présent psychologique en tant que zone de réaction cognitive dans la sélection ou le rejet des formes linguistiques (temps verbaux, phrases nominales, interjections).

Mais, en creux, un autre objectif apparaissait : se servir des genres de discours, de leur constitution, de leur rapport au monde réel, pour affiner la connaissance des faits de langue pour eux-mêmes. C'est ainsi que ces commentaires sportifs en direct ont mis en lumière ou confirmé en les amplifiant, parce que le genre s'y prêtait, des potentialités ou des contraintes concernant l'infinitif, le futur, le présent prospectif, le passé récent, les noms propres. On peut penser que le recours systématique à des observations faites à partir de multiples genres de discours pourrait améliorer notre connaissance de la langue. D'une certaine façon, ce serait réduire la distance entre la langue et le discours.

\section{Références bibliographiques}

Adam J-M. (2001). Types de textes ou genres de discours? Comment classer les textes qui disent de et comment faire?, Langages $n^{\circ} 141$. pp.10-27.

Bakthine M. (1984). Les genres de discours. Esthétique de la création verbale. Paris : Gallimard. Barcelo J. et Bres J.

(2006). Les temps de l'indicatif. Paris : Ophrys.

Bres J. (2005). Le présent de l'indicatif en français : de quelques problèmes, et peut-être de quelques solutions, Desepierres $\mathrm{Cl}$ et Krazem M. (eds). Le présent de l'indicatif. Dijon : Centre Bachelard,

Bres J. (2007). Le discours médiatique sportif aux prises avec le temps verbal, in Le français parlé dans les médias. Actes du colloque de l'Université de Stockholm, 8-12 juin 2005, M. Broth, M. Forsgren, C. Norén et F. SulletNylander (éd.), Stockolm : Acta Universitatis stockholmiensis, pp- 83-96.

Biber D. (1988). Variation across speach and writing. Cambridge : University Press Bonnard H. (1982). Code du français courant. Paris : Magnard,.

Confais J.-P. (1990). Temps, mode, aspect. Toulouse : P.U.M

Co V. (1980). La notion de monde possible et le système temporel et aspectuel du français. Langages $n^{\circ}$ 64. pp109-124

Deulofeu, J. (2000). Les commentaires sportifs constituent-ils un genre au sens linguistique du terme ?. Corpus. Méthodologie et applications linguistiques, Bilger, M (ed). Paris : Champion. Pp271-295

Gosselin, L. (1996). Sémantique de la temporalité en français. Bruxelles : Duculot

Grinshpun Y. et Krazem M. (2005). Quelques marqueurs linguistiques liés au présent de l'indicatif dans les commentaires sportifs en direct. Le présent de l'indicatif Despierres C et Krazem M (eds). Centre Bachelard : Dijon. Pp117-142

Guillaume G. (1929). Temps et verbe. Paris : Champion

Krazem, M. (2005). Le présent sportif. Le présent de l'indicatif. Despierres C et Krazem M (eds). Dijon : entre Bachelard pp-77-117 Krazem, M. (2008). Le présent « actuel » pour décrire trois corpus singuliers : le bon choix

didactique ?. Acquisition et didactique 1 Heather Hilton (ed). Chambéry : PUS. pp-83-98

Krazem, M. (2011a). Représenter les relations entre grammaire et genres de discours : 1'exemple des commentaires sportifs . LINX $n^{\circ} 64-65$. pp45-68

Krazem M. (2011b). Zidane, qui passe à Sganarelle, qui lance Platini...ou comment des noms propres deviennent phrases averbales.

Les énoncés averbaux autonomes entre grammaire et discours, F.Lefeuvre et I.Behr (éds). Paris : Ophrys. pp-121-

$136 \mathrm{Lab}$, F. et Le Goffic P. (2001). Le présent pro futuro. Cahiers Chrono n 7. pp-77-98

Martine J. et Rose D. (2008). Genre relations. Mapping culture. London: Equinox. 
Mathon C et Boulakia G. (2011). « Le commentaire sportif en direct : une combinatoire de différentes fonctions de la prosodie », Yoo, H-Y \& Delais-Roussarie, E. (eds). Actes d'IDP 2009. Paris, 9-11 septembre 2009.

Nef F. (1986). Sémantique de la référence temporelle en français moderne. Berne : Peter Lang, Rabatel, A. (1998). La construction textuelle du point de vue. Lausanne : Delachaux et Niestlé

Serbat G. (1980). La place du présent de l'indicatif dans le système des temps. L'information grammaticale, $n^{\circ}$. pp-3639

Serbat, G. (1988). Le prétendu présent de l'indicatif : une forme non-déictique du verbe. L'information grammaticale, $n^{\circ}$ 38. pp-32- 35

Wilmet M. (1980). Le temps linguistique, L'information grammaticale, $n^{\circ} 38$. pp-6-10 Wilmet M. (1997). Grammaire critique du français. Bruxelles : Duculot

\footnotetext{
${ }^{1}$ Dans la lignée notamment des travaux de Biber (1988), Martin et Rose (2008). Je profite de cette note pour remercier les relecteurs pour leurs remarques.

${ }^{2} \mathrm{~J}$ 'ai réuni seul le corpus avec lequel je travaille. Celui-ci est très large, autant oral qu'écrit (journaux, internet, sous-

titrages malentendants). Pour la partie qui concerne cette contribution, le corpus est constitué d'enregistrements radio en direct de matchs de football ou de multiplexes, capturés par vagues successives au gré d'études antérieures sur ces questions (2004-2005, 2009-2012), d'enregistrements de matchs télévisés dans les mêmes périodes (avec quelques enregistrements de rugby). Beaucoup de données télévisées datant de la coupe du monde 1998 sont utilisées.

Les jeux olympiques de Pékin, de Londres et marginalement de Rio ont élargi les enregistrements à d'autres sports. Une part importante du corpus est constitué d'enregistrements et de textes traitant du cyclisme à cause de la dimension narrative marquée des commentaires de ce sport (période 2001-2019). A cela s'ajoutent des énoncés saisis « au vol », particulièrement les infinitifs « injonctifs » oraux.

${ }^{3}$ Voir Krazem (2011a) pour l'importance des noms de genres dans leur perception comme objets linguistiques.

${ }^{4}$ Je renvoie pour une présentation synthétique à Bres (2005) et Krazem (2008). En allant vite : présent déictique, présent actuel, présent atemporel.

${ }^{5}$ Le terme «présent » appliqué aux temps verbaux recoupe, on le sait, des réalités très différentes.

${ }^{6} \mathrm{Il}$ semble que ce j'appelle « captation » dans la zone du présent psychologique corresponde au moins en partie à la

« saisie » guillaumienne dans le « temps opératif» (Guillaume 1929). Mais n’ayant pas été formé dans ce cadre théorique, je ne suis pas certain du degré de correspondance. Je préfère utiliser le terme « captation » plutôt que celui de

« saisie ».

${ }^{7}$ Cette terminologie (Barcelo et Bres 2006) a l'avantage selon moi d'éviter les amalgames trop fréquents entre le futur simple et la forme en aller +infinitif. Les commentaires sportifs montrent bien la différence entre les deux.

${ }^{8}$ Nous renvoyons à Mathon et Boulakia (2011) pour une analyse plus fine de la prosodie des CS télé en direct.

${ }^{9}$ Originalité qui a des points communs avec les noms propres des didascalies (Krazem 2011b)

${ }^{10}$ Ainsi, un « long ballon » n'est pas un ballon de forme longue mais une longue passe.

${ }^{11}$ Deulofeu (2000) relève un élément crucial : le nom propre est pris dans un noyau caractérisable par des marques aspectuo-temporelles (itération mais vue dans une perspective de succession temporelle). Alors que l'exemple (a) est régulièrement attesté, la construction (b) est exclue.
}

(a) Re Casoni pied gauche loin devant

(b) *re-Casoni frappe le corner

${ }^{12}$ Seul le pronom relatif « qui » est observé. Voir Krazem (2011b) pour une comparaison avec les noms de personnage dans les didascalies où tous les pronoms relatifs sont observés. C'est le recul quasi nul qui explique l'absence d'autres relatifs.

${ }^{13}$ Soit dit en passant le sémantisme de l'expression «tenter sa chance », au passé et même au présent, est le signe que le tir n'a pas abouti au moment du début de l'intervalle de l'énonciation.

${ }^{14}$ Voir par exemple Lab et Le Goffic (2002). Le présent montre les faits comme certains tandis que le futur laisse une zone d'incertitude. 
${ }^{15}$ Toutefois, ces infinitifs oraux ne sont pas endémiques aux commentaires sportifs. On en trouve ailleurs, plus rarement :

(a) c'est vrai (mais) ne pas oublier que, encore une fois, ce n'est pas la tradition par moment qui compte c'est la transmission des choses » (Thierry Marx, chronique France info)

(b) « Surtout ne pas arriver en retard demain » (pris au vol) 